CERN-TH/99-157

hep-ph/9905545

\title{
Heavy quark photoproduction [
}

\section{Stefano Frixione ${ }^{\mathrm{a}}$

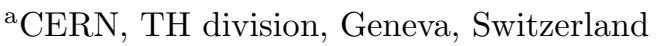 collisions. \\ 1. Computation of cross sections at fixed order in QCD}

I present a review of selected topics in the computation of heavy flavour cross sections in photon-hadron

A heavy quark $Q$ is by definition a quark whose mass $m_{Q}$ is much larger than $\Lambda_{Q C D}$. Since $m_{Q}$ is different from zero, it cuts off the singularities due to the collinear emission of gluons from the heavy quark lines, thus allowing the definition of open-heavy-quark cross sections, that are infrared-finite order by order in perturbative QCD. The hard scale of the production process is of the order of $m_{Q}$. Therefore, one expects the perturbative predictions to be very reliable in the case of top, under control in the case of bottom, and affected by large uncertainties in the case of charm. This is more so, since the smaller the mass, the bigger the impact that effects of non-perturbative origin will have on the predictions of physical observables. The cross section for the production of $Q \bar{Q}$ pairs in on-shell photonhadron reactions is usually written as the sum of two terms:

$$
\begin{aligned}
\sigma_{\gamma H} & =f_{j}^{(H)}\left(\mu_{F}\right) \otimes \hat{\sigma}_{\gamma j}\left(\mu_{R}, \mu_{F}, \mu_{\gamma}\right) \\
& +f_{i}^{(\gamma)}\left(\mu_{\gamma}\right) \otimes f_{j}^{(H)}\left(\mu_{F}^{\prime}\right) \otimes \hat{\sigma}_{i j}\left(\mu_{R}^{\prime}, \mu_{F}^{\prime}, \mu_{\gamma}\right)
\end{aligned}
$$

denoted as pointlike and hadronic components respectively. Neither of the two is well defined beyond LO in perturbation theory. This fact is formally taken into account in eq. (11) by the dependence upon the scale $\mu_{\gamma}$, which is related to the subtraction of singularities due to the collinear splitting of the incoming photon. The pointlike and hadronic components are not constant with respect to a variation of $\mu_{\gamma}$, while their sum is (up to higher order terms in perturbation theory). On the other hand, the pointlike and hadronic com-

\footnotetext{
${ }^{1}$ Talk given at DIS99, $19-23$ April 1999, Zeuthen, DE.
}

ponents are separately constant with respect to a variation of all the other mass scales which enter eq. (11). The contribution of the hadronic component will be larger (relative to the pointlike component) the smaller $m_{Q}$. However, it has to be remarked that kinematical configurations exist where the hadronic component is basically negligible: typical examples are the high- $p_{T}$ region of the emitted heavy quarks, and small center-ofmass energy collisions (like those at fixed-target experiments). This is due to the fact that photon densities $f_{i}^{(\gamma)}(x)$ are vanishing faster than hadron densities for $x \rightarrow 1$.

The task for theorists is to calculate the partonic cross sections $\hat{\sigma}_{\gamma i}$ and $\hat{\sigma}_{i j}$ at the highest possible order in perturbation theory. In ref. [1] (for the pointlike component) and in ref. [2] (for the hadronic component) results accurate to NLO ( $\alpha_{e m} \alpha_{S}^{2}$ and $\alpha_{S}^{3}$ respectively) have been obtained. At that time, predictions were only available for total cross sections and single-inclusive spectra. Later, computations were performed [ 3], which allowed the definition of cross sections fully exclusive in terms of the variables of $Q, \bar{Q}$, and of the accompanying jet. The matrix elements relevant for $Q \bar{Q}$ production are mostly convenient calculated in a modified $\overline{\mathrm{MS}}$ scheme [ [4], in which the renormalized $\alpha_{S}$ is defined through the following equation (up to terms of order $\alpha_{S}^{3}$ ):

$$
\begin{aligned}
\alpha_{S}^{\text {bare }} & =\mu^{2 \epsilon} \alpha_{S}^{r e n}\left[1-\frac{\alpha_{S}^{r e n}}{4 \pi \bar{\epsilon}} \frac{11 C_{A}-4 T_{F} N_{l f}}{3}\right] \\
& \times\left[1+\frac{\alpha_{S}^{\text {bare }}}{4 \pi \bar{\epsilon}} \frac{4 T_{F} m_{Q}^{-2 \epsilon}}{3}\right]
\end{aligned}
$$

where $N_{l f}$ is the number of flavours lighter than $Q$ (i.e., 3 for charm, 4 for bottom and 5 for top). The first term in square brackets in eq. (2) is the 
same that appears in the standard $\overline{\mathrm{MS}}$ scheme; thus, light flavours are dealt with like in standard $\overline{\mathrm{MS}}$. The second term in square brackets is on the other hand peculiar of heavy flavour production, and is designed in such a way that, for momenta much smaller than $m_{Q}$, it cancels exactly the contributions of heavy quark divergent loops. Since this second term is only expressed in terms of $\alpha_{S}^{\text {bare }}$, it follows that the renormalization group equation one derives is equal to that of the standard $\overline{\mathrm{MS}}$ with $N_{\text {lf }}$ flavours. This is consistent with the fact that no contribution to the cross section comes from diagrams where a heavy quark is present in the initial state.

The cross sections computed in this way are expected to give sensible predictions for momenta of the order of $m_{Q}$. At the borders of the phase space, or for high energies, some logarithmic terms may grow large and spoil the convergence of the perturbative series. Example of large logarithms are: $\log \left(S / m_{Q}^{2}\right)$ (small- $x$ effects: they are important when the center-of-mass energy of the colliding particles is large); $\log \left(p_{T}^{Q \bar{Q}} / m_{Q}\right)$ (due to the emission of soft gluons, they are important at the edges of the phase space or at the threshold); $\log \left(p_{T} / m_{Q}\right)$ (due to the emission of collinear gluons from the heavy quark line, they are important when $\left.p_{T} \gg m_{Q}\right)$. It has been shown that the impact of small- $x$ effects for charm and bottom production at present colliders is moderate. Also, soft gluon resummation gives small enhancements with respect to NLO results for total cross sections in the case of top production at the Tevatron and of bottom production at HERA-B. In the following, I will concentrate on collinear logarithms, which are phenomenologically relevant for charm photoproduction at HERA.

\section{The large transverse momentum region}

If $p_{T} \gg m_{Q}$, mass terms in the matrix elements are suppressed by powers of $m_{Q} / p_{T}$. Therefore, when computing matrix elements one is entitled to treat the heavy quark as massless [ 5, 6] and use the standard $\overline{\mathrm{MS}}$ scheme (this is the reason why this kind of calculations are often - incorrectly - referred to as "massless"). This implies that it is mandatory to consider diagrams where the heavy quark is also present in the initial state. Furthermore, the final state $Q g$ collinear singularities are no longer cut off by the quark mass. The subtraction of these additional singularities is performed within the framework of the factorization theorem; one writes the cross section for the production of a heavy quark (in any kind of hard collisions) as follows:

$d \sigma_{Q}=d \sigma_{i}(\mu) \otimes D_{Q}^{(i)}(\mu)$,

where $i$ can be a gluon, a light quark or the heavy quark $Q$, and $d \sigma_{i}$ is the cross section for the production of the flavour $i$. $D_{Q}^{(i)}$ are the so-called perturbative fragmentation functions (PFF), whose bare form contain a divergent term which cancels the final-state collinear singularities mentioned above. As far as the $\mu$ dependence is concerned, the PFFs obey the usual Altarelli-Parisi equations. However, at variance with the case of parton densities, the PFFs are fully calculable in perturbation theory, for scales $\mu=\mu_{0}$ of the order of the heavy quark mass (this prevents $D_{Q}^{(i)}\left(\mu_{0}\right)$ from containing large logarithms). Such a calculation has been performed in ref. [7], up to terms of order $\alpha_{S}^{2}$. At this order, $D_{Q}^{(Q)}$ and $D_{Q}^{(g)}$ are non-vanishing, while $D_{Q}^{(q)}$ is zero. The main feature of eq. (3) is the following: the scale $\mu$ is of the order of $p_{T}$, which is the hard scale of the process. Since in the computation of matrix elements the mass of the heavy quark has been set to zero, $d \sigma_{i}$ does not contain any large logarithm of $p_{T} / m_{Q}$. All the mass effects are included in $D_{Q}^{(i)}$; however, here the large logarithms are resummed by the Altarelli-Parisi equations, in the evolution from the scale $\mu_{0}$ to the scale $\mu$.

If the data are presented in terms of heavyflavoured hadrons $H_{Q}$, theoretical predictions must be given for such quantities; these are calculated starting from the heavy-quark cross section given in eq. (3), and convoluting it with a non-perturbative fragmentation function (NPFF), that accounts for the hadronization process $Q \rightarrow H_{Q}$ :

$d \sigma_{H_{Q}}=d \sigma_{Q} \otimes D_{H_{Q}}^{(Q)}$

(of course, the convolution with a NPFF can be also performed starting from a fixed-order 
QCD cross section, like the one in eq. (11)). The NPFFs can not be calculated in perturbation theory; however, they are supposed to be universal. Therefore, they can be determined by a fit to experimental data - typically, from $e^{+} e^{-}$collisions - and eventually used to obtain theoretical predictions for various production processes. Recent fits are presented in refs. [ 8, 9]; it turns out that the NPFF is much harder than in previous determinations based on LO QCD analysis. A different procedure has also been proposed [ 6]. Instead of considering PFFs and NPFFs, one can introduce a fragmentation function which describes at the same time the hard and soft physics: $D_{H_{Q}}^{(i)}=$ $D_{Q}^{(i)} \otimes D_{H_{Q}}^{(Q)}$. The idea is then to parametrize $D_{H_{Q}}^{(i)}$, fit it to the data, and then evolve it to the appropriate scale with Altarelli-Parisi equations. However, since the NPFFs considered in practice do not depend upon a mass scale, there is no difference in evolving only the PFF or the convolution of the PFF and the NPFF (this can be trivially shown by writing the Altarelli-Parisi equations in Mellin space). Therefore, ref. [5] and [6] only differ in the choice of the initial conditions for the evolution of the fragmentation function. While in ref. [5] the $\alpha_{S}$ terms of $D_{Q}^{(i)}\left(\mu_{0}\right)$ are taken into account, they are not included in ref. [6]. Thus, ref. [6] only includes mass effects through a fitting to data, and does not exploit all the perturbative results available.

\section{Matching large and small momentum results}

As discussed in the previous sections, if $p_{T}$ is of the order of $m_{Q}$, then fixed-order QCD computations have to be used; on the other hand, if $p_{T}$ is much larger than $m_{Q}$, resummed calculations are mandatory. What is missing, unfortunately, is a quantitative definition of what "much larger" means. HERA data are compared to resummed calculations for $p_{T}\left(D^{*}\right)$ down to $2 \div 3 \mathrm{GeV}$, which means $p_{T}(c)$ on average smaller than $4 \mathrm{GeV}$ (here and in what follows, I take $p_{T}\left(D^{*}\right)=\langle z\rangle p_{T}(c)$, where $\langle z\rangle$ is the average $z$ obtained using a Peterson fragmentation function with $\epsilon=0.03)$. The ratio $4 \mathrm{GeV} / m_{c}$ does not seem to be large enough.
Any agreement between data and resummed cross sections in this $p_{T}$ region should therefore be regarded as incidental. However, it is clear that there is an intermediate region in $p_{T}$ where both fixed-order and resummed calculations are almost equally reliable (or, if one adopts a pessimistic point of view, neither of the two is meaningful). Therefore, it is conceivable to have a matched calculation, which returns a fixed-order result at low $p_{T}$, a resummed result at large $p_{T}$, and smoothly connects the two approaches in the intermediate region. Unfortunately, such a calculation does not exist for photoproduction. However, results are available for hadroproduction [ 10, 11]. It is instructive to see how this matter is dealt with in ref. [10], since there is no conceptual difference with respect to the case of photoproduction (to recover the latter, simply substitute $\alpha_{S}^{n}$ with $\alpha_{e m} \alpha_{S}^{n-1}$ in the following equations). The fixed (NL)-order and (NLL) resummed cross sections are written as ( $\mu$ is a scale of the order of $\left.p_{T}\right)$ :

$$
\begin{aligned}
\left(\frac{d \sigma}{d p_{T}^{2}}\right)_{N L O} & =\alpha_{S}^{2} A+\alpha_{S}^{3} B+\mathcal{O}\left(\alpha_{S}^{4}\right) \\
\left(\frac{d \sigma}{d p_{T}^{2}}\right)_{R E S} & =\alpha_{S}^{2} \sum_{n=0}^{\infty} a_{n}\left(\alpha_{S} \log \frac{\mu}{m_{Q}}\right)^{n} \\
& +\alpha_{S}^{3} \sum_{n=0}^{\infty} b_{n}\left(\alpha_{S} \log \frac{\mu}{m_{Q}}\right)^{n} \\
& +\mathcal{O}\left(\alpha_{S}^{4}\left(\alpha_{S} \log \frac{\mu}{m_{Q}}\right)^{n}\right)
\end{aligned}
$$

The coefficients $A$ and $B$ contain logarithms of $p_{T} / m_{Q}$. Eq. (6) is defined up to power-suppressed terms, not explicitly indicated. We can now define the matched cross section:

$$
\begin{aligned}
\frac{d \sigma}{d p_{T}^{2}} & =\alpha_{S}^{2} A+\alpha_{S}^{3} B+\left\{\alpha_{S}^{2} \sum_{n=2}^{\infty} a_{n}\left(\alpha_{S} \log \frac{\mu}{m_{Q}}\right)^{n}\right. \\
& \left.+\alpha_{S}^{3} \sum_{n=1}^{\infty} b_{n}\left(\alpha_{S} \log \frac{\mu}{m_{Q}}\right)^{n}\right\} G\left(m_{Q}, p_{T}\right),(7)
\end{aligned}
$$

where the function $G$ is arbitrary to a large extent, but must approach 1 when $m_{Q} / p_{T} \rightarrow 0$. If $p_{T} \simeq m_{Q}$, the quantity in curly brackets vanishes, and the matched cross section approaches eq. (5). If $p_{T} \gg m_{Q}$, the matched cross section approaches eq. (6), since logarithmic terms in $A$ 
and $B$ dominate (by construction, these terms are exactly those missing in the sums of eq. (7) with respect to the sums of eq. (6)). Notice that this is true regardless of the form of $G$, provided that it satisfies the condition given above. One could therefore choose $G \equiv 1$. However, as shown in ref. [ 10], this choice can lead to instabilities in the numerical computations. In ref. [10], the following form has been adopted:

$G\left(m_{Q}, p_{T}\right)=\frac{p_{T}^{2}}{p_{T}^{2}+\beta^{2} m_{Q}^{2}}$,

where $\beta$ is a suitable number. From numerical studies performed for bottom production at the Tevatron, it turns out that a sensible choice is $\beta=5$. This means that, in the matched cross section, the whole tower of logarithms (except those with coefficients $a_{0}, a_{1}$ and $b_{0}$ ) is suppressed by a factor 0.5 for $p_{T}=5 m_{Q}$, and is almost fully included $(G=0.9)$ for $p_{T}=15 m_{Q}$. If we assume that these conclusions can be also applied to charm production at HERA, we conservatively conclude that a resummed cross section can be safely compared to the data starting from a $p_{T}(c)$ of the order of $22 \mathrm{GeV}$ (here, $m_{c}=1.5 \mathrm{GeV}$ ), that is for $p_{T}\left(D^{*}\right)$ of the order of $16 \mathrm{GeV}$. Being less conservative, we can say that the lowest $p_{T}(c)$ value for which a comparison between the data and a resummed cross section is meaningful is of the order of $8 \mathrm{GeV}\left(p_{T}\left(D^{*}\right)\right.$ of the order of $5 \div 6 \mathrm{GeV})$.

\section{Conclusions}

I presented a review of results relevant for the production of heavy flavours in high-energy photon-hadron collisions. At low and moderate transverse momenta, where the bulk of the total cross section comes from, fixed-order QCD computations (available to NLO accuracy) are appropriate. HERA data on charm production have renewed interest in the resummation of large logarithms of the transverse momentum. A brief discussion on the techniques designed to perform this resummation has been given. Finally, it has been argued that most of the currently available data for $D^{*}$ production at HERA have too small a $p_{T}$ to be safely compared with a resummed cal- culation. A definite statement on this issue will however only come when a matched calculation will be available, which combines the results of fixed-order and resummed computations.

Acknowledgements: I would like to thank Matteo Cacciari for useful discussions.

\section{REFERENCES}

1. R.K. Ellis and P. Nason, Nucl. Phys. B312(1989)551; J. Smith and W.L. van Neerven, Nucl. Phys. B374(1992)36.

2. P. Nason, S. Dawson and R.K. Ellis, Nucl. Phys. B303(1988)607; P. Nason, S. Dawson and R.K. Ellis, Nucl. Phys. B327(1989)49; (E:ibid. B335(1990)260); W. Beenakker, H. Kuijf, W.L. van Neerven and J. Smith,Phys. Rev. D40(1989)54; R. Meng, G.A. Schuler, J. Smith and W.L. van Neerven, Nucl. Phys. B339(1990)325; W. Beenakker, W.L. van Neerven, R. Meng, G.A. Schuler and J. Smith, Nucl. Phys. B351(1991)507.

3. M.L. Mangano, P. Nason and G. Ridolfi, Nucl. Phys. B373(1992)295; S. Frixione, M.L. Mangano, P. Nason and G. Ridolfi, Nucl. Phys. B412(1994)225.

4. J. Collins, F. Wilczek and A. Zee, Phys. Rev. D18(1978)242; W.J. Marciano, Phys. Rev. D29(1984) 580

5. M. Cacciari and M. Greco, Nucl. Phys. B421(1994)530; M. Cacciari and M. Greco, Z. Phys. C69(1996) 459.

6. B.A. Kniehl, G. Kramer and M. Spira, Z. Phys. C76(1997)689; J. Binnewies, B.A. Kniehl and G. Kramer, Z. Phys. C76(1997)677; J. Binnewies, B.A. Kniehl and G. Kramer, Phys. Rev. D58(1998)014014.

7. B. Mele and P. Nason, Nucl. Phys. B361(1991)626.

8. P. Nason and C. Oleari, hep-ph/9903541.

9. M. Cacciari and M. Greco, Phys. Rev. D55(1997)7134.

10. M. Cacciari, M. Greco and P. Nason, JHEP 9805(1998)007.

11. F.I. Olness, R.J. Scalise and W.K. Tung, Phys. Rev. D59(1999)014506. 